ARTICLE

https://doi.org/10.1038/s41467-019-09872-w

\title{
Ultrafast charge transfer coupled with lattice phonons in two-dimensional covalent organic frameworks
}

Tae Wu Kim (10) 1,2,3,8, Sunhong Jun 3,9, Yoonhoo Ha (1) 1, Rajesh K. Yadav ${ }^{4}$, Abhishek Kumar (D) ${ }^{4}$

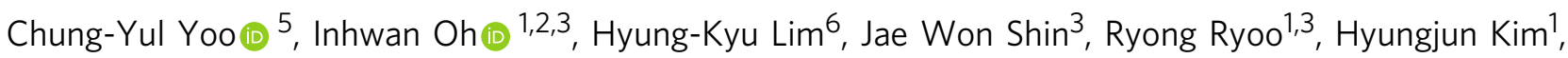
Jeongho Kim (1) ${ }^{7}$, Jin-Ook Baeg (1) ${ }^{4} \&$ Hyotcherl Ihee (i) ${ }^{1,2,3}$

Covalent organic frameworks (COFs) have emerged as a promising light-harvesting module for artificial photosynthesis and photovoltaics. For efficient generation of free charge carriers, the donor-acceptor (D-A) conjugation has been adopted for two-dimensional (2D) COFs recently. In the 2D D-A COFs, photoexcitation would generate a polaron pair, which is a precursor to free charge carriers and has lower binding energy than an exciton. Although the character of the primary excitation species is a key factor in determining optoelectronic properties of a material, excited-state dynamics leading to the creation of a polaron pair have not been investigated yet. Here, we investigate the dynamics of photogenerated charge carriers in 2D D-A COFs by combining femtosecond optical spectroscopy and non-adiabatic molecular dynamics simulation. From this investigation, we elucidate that the polaron pair is formed through ultrafast intra-layer hole transfer coupled with coherent vibrations of the 2D lattice, suggesting a mechanism of phonon-assisted charge transfer.

\footnotetext{
${ }^{1}$ Department of Chemistry, Korea Advanced Institute of Science and Technology (KAIST), Daejeon 34141, Republic of Korea. ${ }^{2}$ KI for the BioCentury, Korea Advanced Institute of Science and Technology (KAIST), Daejeon 34141, Republic of Korea. ${ }^{3}$ Center for Nanomaterials and Chemical Reactions, Institute for Basic Science (IBS), Daejeon 34141, Republic of Korea. ${ }^{4}$ Artificial Photosynthesis Research Group, Korea Research Institute of Chemical Technology (KRICT), Daejeon 34114, Republic of Korea. ${ }^{5}$ Korea Institute of Energy Research (KIER), Daejeon 34129, Republic of Korea. ${ }^{6}$ Department of Chemical Engineering, Kangwon National University, Gangwon-do 24341, Republic of Korea. ${ }^{7}$ Department of Chemistry, Inha University, Incheon 22212 , Republic of Korea. ${ }^{8}$ Present address: Chemical Sciences and Engineering Division, Argonne National Laboratory, Lemont, Illinois 60439, USA. ${ }^{9}$ Present address: Memory Business, Samsung Electronics, Gyeonggi-do 18448, Republic of Korea. Correspondence and requests for materials should be addressed to H.K. (email: linus16@kaist. ac.kr) or to J.K. (email: jkim5@inha.ac.kr) or to J.-O.B. (email: jobaeg@krict.re.kr) or to H.I. (email: hyotcherl.ihee@kaist.ac.kr)
} 
C ovalent organic frameworks (COFs) with periodically ordered architecture have attracted much interest as a promising functional material for artificial photosynthesis and photovoltaic devices. In COFs, organic molecules are covalently bonded to each other to form two-dimensional (2D) or three-dimensional porous crystalline structures ${ }^{1-6}$. Specifically, $2 \mathrm{D}$ COFs consist of $\pi$-conjugated aromatic molecules forming a 2D layer and the 2D layers stack up on top of each other to form a three-dimensional structure. Electronic interaction among the building blocks of a $2 \mathrm{D} \mathrm{COF}$ and inter-layer $\pi-\pi$ interaction among the stacked layers of COFs provide efficient pathways for photogenerated charge carriers. Due to their robust structure and high charge mobility through the framework, the COFs have the potential to serve as a primary light-harvesting module in photovoltaic and optoelectronic devices ${ }^{2-5,7-10}$.

In artificial photosynthetic systems such as organic polymer solar cells, photons are converted into electrical energy via a series of steps ${ }^{1-17}$. First, photons are absorbed by chromophores to generate charge carriers bound by Coulombic interaction (i.e., excitons). Then, the excitons diffuse toward a heterojunction, e.g., the polymer/fullerene interface, where an exciton is separated into free charge carriers (i.e., an electron and a hole). This process of charge separation in artificial light-harvesting systems occurs on ultrafast time scales ranging from tens of femtoseconds ( $f$ ) to several picoseconds ${ }^{12-16}$, as commonly observed in natural photosynthesis ${ }^{18-20}$.

For excitons to be separated into free charge carriers, the exciton-binding energy should be overcome. In this regard, for high-performance solar cells, it is desirable to have a lightharvesting system where excitations of low exciton-binding energy are generated. As a means of lowering the excitonbinding energy, researchers have recently developed 2D COFs consisting of two types of repeat units with different electron affinities ${ }^{7,8,21,22}$, borrowing the concept of low-bandgap polymers employing a donor-acceptor (D-A) conjugation scheme ${ }^{12,14}$. In those D-A COFs, the repeat units with low and high electron affinities can serve as an electron donor and an electron acceptor, respectively. As a result of the D-A conjugation, photoexcitation of $2 \mathrm{D} \mathrm{D}-\mathrm{A}$ COFs would lead to the formation of a polaron pair, which is a precursor to free charge carriers and has lower binding energy than an exciton, as in the low-bandgap D-A copolymers. Although the character of the primary excitation species is crucial for determining optoelectronic properties of a material, excitedstate dynamics leading to the creation of a polaron pair in the $2 \mathrm{D}$ $\mathrm{D}-\mathrm{A}$ COFs have not been investigated yet. According to previous studies of photoinduced charge carrier dynamics in D-A $\mathrm{COFs}^{21,22}$, it was suggested that light absorption induces charge transfer between each donor and acceptor units within $1.8 \mathrm{ps}$ and the charges stay separated for up to $10 \mu$ s due to charge delocalization in two separate donor and acceptor arrays aligned perpendicular to stacked 2D layers. This result shows a unique molecular configuration of D-A COFs where the D-A interfaces serve as a well-ordered columnar heterojunction throughout the three-dimensional framework.

In this work, by applying both time-resolved spectroscopic experiment and theoretical simulation, we investigate the earliest stage (on sub-ps time scale) of charge carrier dynamics in $2 \mathrm{D} \mathrm{D}-\mathrm{A}$ COFs consisting of 3,4,9,10-perylenetetracarboxylic acid diimide (PDI) and free-base porphyrin. As the two constituent units have different electron affinities from each other, the 2D D-A COFs contain the $\mathrm{D}$-A conjugation through $\pi$-electronic interactions between the building blocks. To elucidate the dynamics of photogenerated charge carriers in the $2 \mathrm{D} \mathrm{D}-\mathrm{A}$ COFs, fs-transient absorption (fs-TA) spectroscopy and non-adiabatic molecular dynamics (NA-MD) simulation are employed in this work. From the fs-TA measurement, we find that excited charge carriers are transferred between PDI and free-base porphyrin on ultrafast time scale with 124 fs time constant, leading to the formation of polaron pairs in the 2D lattice of COFs. From the systematic theoretical simulation based on time-dependent density functional theory (DFT), the ultrafast charge transfer can be specifically assigned to the migration of holes from PDI to free-base porphyrin unit. In addition, we demonstrate that this intra-layer charge transfer is coupled with lattice vibrations manifested as coherent oscillations of TA spectra, which is a compelling evidence for a mechanism of phonon-assisted charge transfer in the periodically ordered structure of COFs.

\section{Results}

Femtosecond transient absorption spectroscopy. Figure 1a shows the molecular structure of synthesized COFs composed of $\mathrm{PDI}$ and free-base porphyrin (i.e., PDI-porphyrin COFs). The 2D D-A COFs were synthesized by direct polycondensation of the building blocks and the details for the synthesis and characterization of the COFs are described in Methods, Supplementary Notes 1-3, and Supplementary Figs. 1-5. The porous structure of COFs was characterized by transmission electron microscopy (TEM), nitrogen isotherm, and powder X-ray diffraction (PXRD). The spacing between 2D layers is clearly visualized by a selected area electron diffraction image and the line spacing was determined to be $0.330 \mathrm{~nm}$ as shown in Supplementary Fig. 1. The pore size distribution from the nitrogen isotherm measurement shows a peak at $3.9 \mathrm{~nm}$, which is similar to the distance between the farthest perylene moieties (along the diagonal) of the smallest rectangle unit in a 2D layer. High-resolution-TEM (HR-TEM) images show $1.5 \mathrm{~nm}$ and $1.0 \mathrm{~nm}$ line spacings, which correspond to (200) or (020), and (220) planes, respectively. As discussed in the Supplementary Note 2, HR-TEM data support the staggered structure along the $c$-axis with slipping along $a$-axis and/or $b$-axis. Moreover, the PXRD pattern is also more consistent with the staggered structure as shown in Fig. 1a. The absorption spectrum of PDI-porphyrin COFs dispersed in $\mathrm{N}, \mathrm{N}$-dimethylformamide (DMF) has absorption features in the entire visible region, including vibronic progression, as shown in Fig. 1b and Supplementary Fig. 6. The intense absorption peak at $424 \mathrm{~nm}$ wavelength is mainly attributed to the B state (or Soret band) of freebase porphyrin unit, which has an absorption peak at $419 \mathrm{~nm}$. The broad absorption from 470 to $700 \mathrm{~nm}$ with the peaks at 484, $518,560,592$, and $652 \mathrm{~nm}$ can be assigned to the absorption arising from the electronic interaction between the $S_{0} \rightarrow S_{1}$ transition of PDI (peaked at 481 and $516 \mathrm{~nm}$ ) and the $\mathrm{Q}_{\mathrm{y}}$ and $\mathrm{Q}_{\mathrm{x}}$ transitions of free-base porphyrin (peaked at 513, 548, 590, and $646 \mathrm{~nm}$ ). The electronic interaction between PDI and free-base porphyrin units is manifested as spectral red shift and broadening of the absorption spectrum of COFs compared with the absorption spectrum of free-base porphyrin, as shown in Fig. $1 \mathrm{~b}$ and Supplementary Fig. 6.

To investigate the dynamics of excited charge carriers of COFs dispersed in DMF, we performed fs-TA measurement. For excitation of COFs, we used the $40 \mathrm{fs}$ pulses of $523 \mathrm{~nm}$ center wavelength with the bandwidth of $20 \mathrm{~nm}$ full-width-at-halfmaximum (FWHM). This photoexcitation condition is predominantly resonant with the electronic transition of PDI units, as supported by the theoretical absorption spectrum calculated by time-dependent DFT calculation that will be discussed later. TA spectra, $-\Delta T / T(\lambda, t)$, are shown in Fig. 2a. The ground-state bleaching (GSB) and the stimulated emission (SE) contributions are manifested as negative spectral features from 500 to $560 \mathrm{~nm}$, and the excited-state absorption (ESA) contribution is seen as a broad positive feature in the $560-710 \mathrm{~nm}$ region. According to previous studies ${ }^{23-25}$, the broad ESA band of free-base porphyrin 

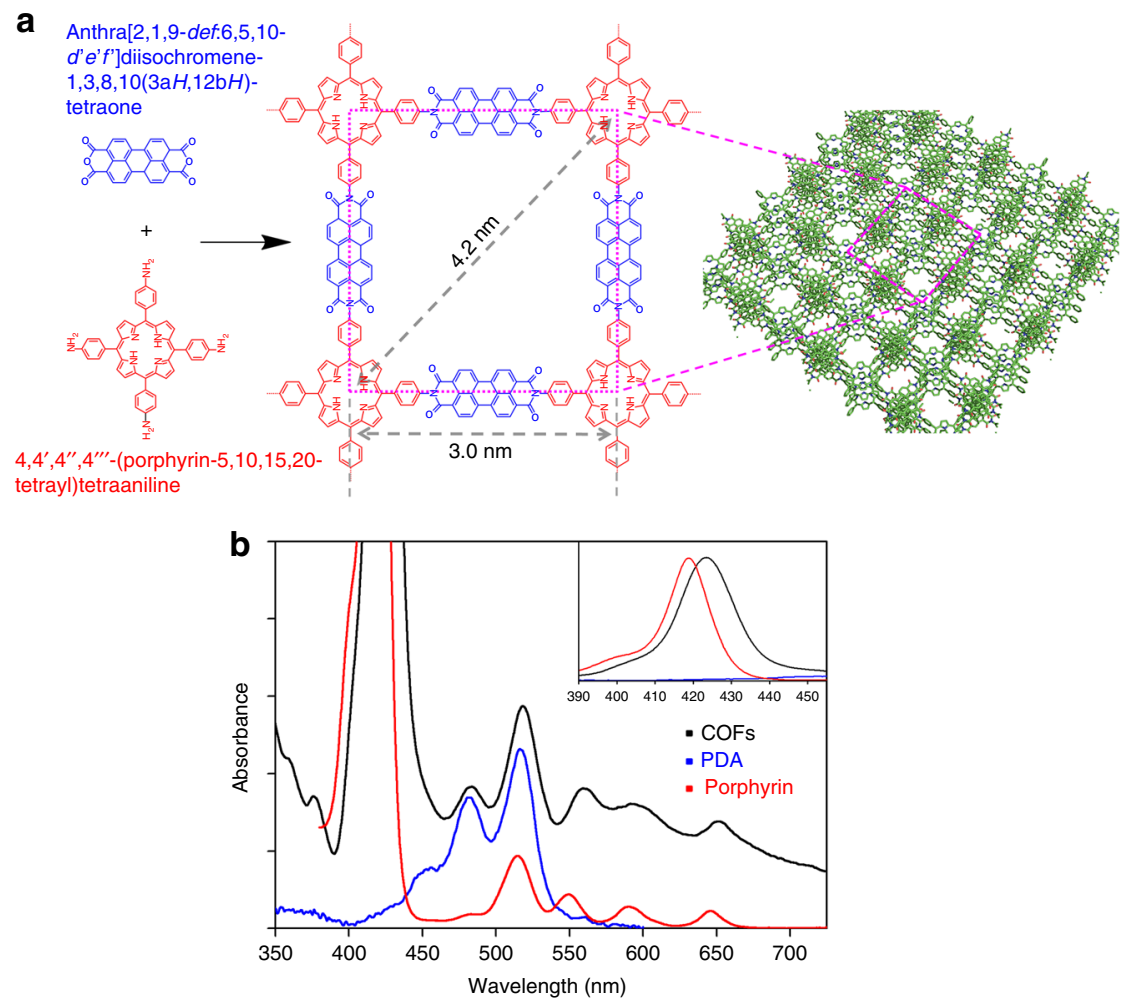

Fig. 1 Molecular structure and steady-state absorption spectra. a Molecular structure of two-dimensional covalent organic frameworks (COFs). For the clear visualization, the repeat units in 2D sheets are truncated from the stacked structure of COFs (green skeleton). The COFs consist of two units, 3,4,9,10-perylenetetracarboxylic acid diimide (PDI) and free-base porphyrin, which are colored in blue and red, respectively. $\mathbf{b}$ Electronic absorption spectrum of PDI-porphyrin COFs dispersed in DMF (black), together with the normalized absorption spectra of their precursor molecules, free-base porphyrin (red), and perylene-3,4,9,10-tetracarboxylic dianhydride called as PDA (blue)

overlaps with the SE of PDI, making it difficult to resolve the spectral features of porphyrin and PDI in the one-dimensional pump-probe spectrum. To overcome the spectral congestion, we performed global kinetic analysis of the TA spectra based on singular value decomposition (SVD). The details of the global kinetic analysis are described in the Supplementary Note 4 and Supplementary Fig. 7. Based on the result of SVD analysis, we applied a sequential kinetic model as follows:

$$
\mathrm{A} \stackrel{124 \mathrm{fs}}{\longrightarrow} \mathrm{B} \stackrel{1.25 \mathrm{ps}}{\longrightarrow} \mathrm{C}
$$

where $\mathrm{A}$ is an initially excited state, and $\mathrm{B}$ and $\mathrm{C}$ are the excited states involved in the dynamics of excited charge carriers. The spectral features of each state are described by species-associated difference spectra (SADS) obtained from the global kinetic analysis, as shown in Fig. $2 \mathrm{~b}$. The transitions among those states were determined to occur with the time constants of $124( \pm 20)$ fs and $1.25( \pm 0.82)$ ps. As can be seen in Fig. 2b, the first SADS shows strong GSB and SE bands in the spectral region from 490 to $625 \mathrm{~nm}$ and these spectral features are in good agreement with the steady-state absorption/emission spectra (Supplementary Fig. 8) and broadband TA spectrum of PDI monomers reported previously ${ }^{26-28}$. Thus, the A state is likely to be associated with PDI units of 2D COFs. The second SADS compared with the first SADS shows that the transition from A to B accompanies quenching of SE and/or spectral broadening of ESA. The SE at $490-625 \mathrm{~nm}$ is quenched further with the transition from B to C as can be seen in the spectral and temporal changes from the second SADS to third SADS shown in Fig. 2b and Supplementary Fig. 9. The decay of $\mathrm{C}$ state cannot be determined from the global kinetic analysis due to the limited time range of our TA measurement. At the longest time delay of our TA data, the third SADS shows non-zero amplitude, indicating that the ground state is not fully recovered.

To examine the identity of the B state, an additional fs-TA measurement was performed on free-base porphyrin dissolved in DMF. We used the same excitation pulse as the one used for COFs to excite the $\mathrm{Q}_{\mathrm{y}}(1,0)$ transition of free-base porphyrin, of which the absorption spectrum is shown in Supplementary Fig. 10. The global analysis of the TA spectra of free-base porphyrin shows that the first SADS and the second SADS decay with the time constants of $81 \mathrm{fs}$ and $945 \mathrm{fs}$, respectively, as shown in Fig. 2d. The ultrafast decay of the first SADS and concomitant growth of the second SADS mainly involve the amplitude change in the spectral range of $610-710 \mathrm{~nm}$. When comparing the SADS of free-base porphyrin with its steady-state emission spectrum (Supplementary Fig. 8), we can infer that the change of SADS at $610-710 \mathrm{~nm}$ is likely to be associated with the growth of SE contribution arising from the $\mathrm{Q}_{\mathrm{x}}(0,0)$ state of free-base porphyrin, which can be reached by $\mathrm{Q}_{\mathrm{y}}$-to- $\mathrm{Q}_{\mathrm{x}}$ internal conversion and vibrational relaxation, as reported previously $23,29,30$. We note that the spectral feature of the valley shape around $645 \mathrm{~nm}$ in the second SADS of free-base porphyrin (Fig. 2d) was also observed in the second SADS of COFs (Fig. 2b), indicating that the dip at $645 \mathrm{~nm}$ in the second SADS of COFs corresponds to the SE contribution arising from the free-base porphyrin units (Supplementary Fig. 8). Thus, the B state must be associated with porphyrin units of $2 \mathrm{D} \mathrm{COFs}$.

Based on the results obtained from the TA measurements on COFs and free-base porphyrin, in the TA spectra of COFs, the quenching of SE at $490-625 \mathrm{~nm}$ arising from PDI units concurs with the growth of SE at $645 \mathrm{~nm}$ arising from free-base porphyrin 
a

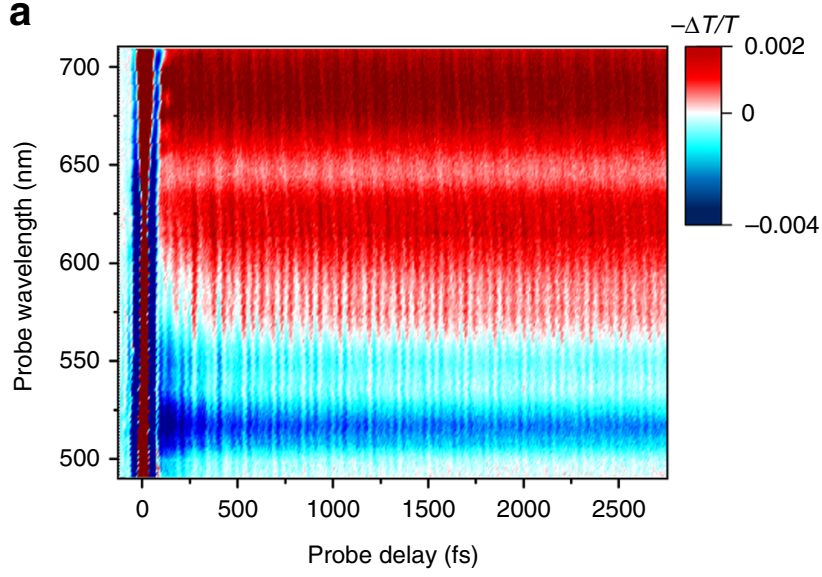

C

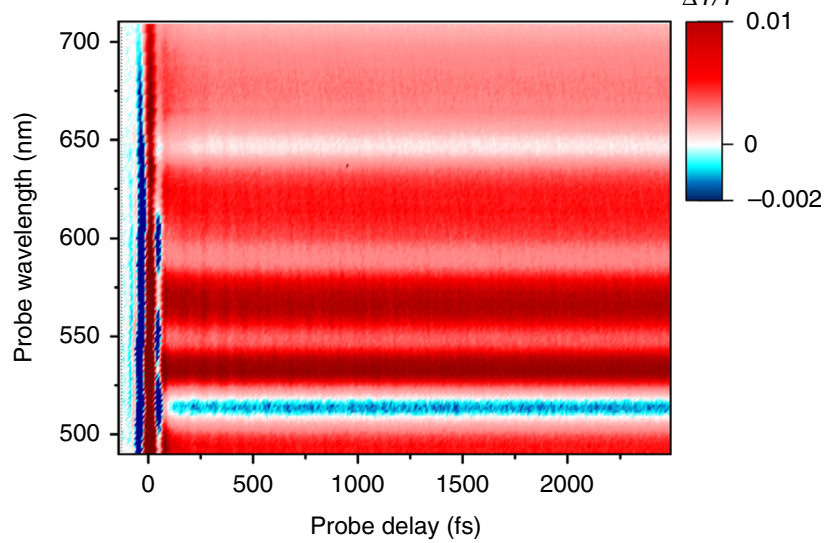

b
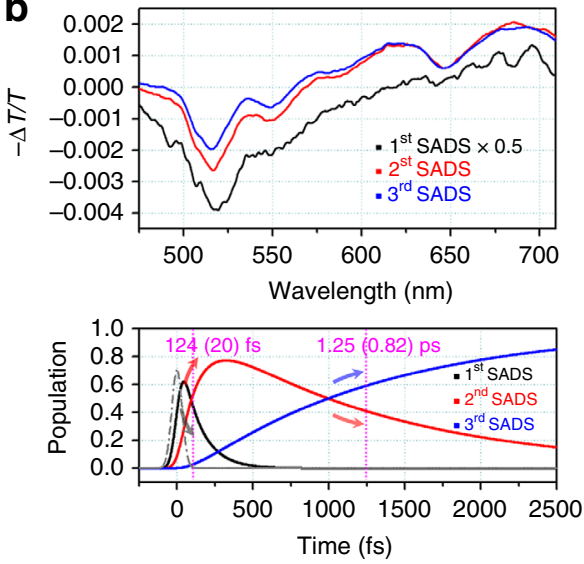

d
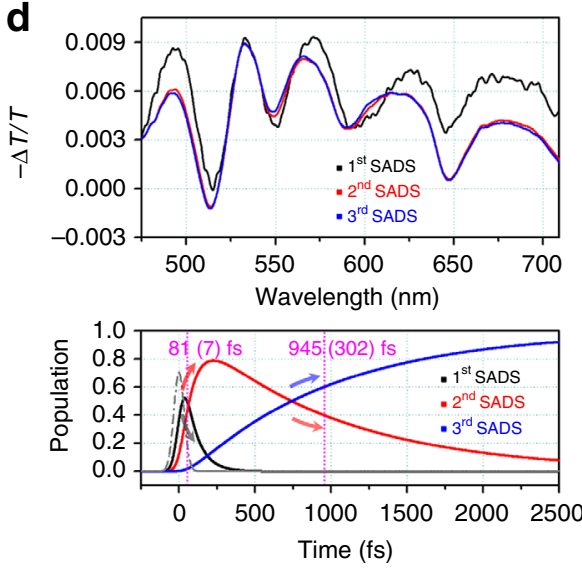

Fig. 2 Two-dimensional transient absorption (TA) spectra. a Broadband TA spectra of COFs. $\mathbf{b}$ The upper panel shows the species-associated difference spectra (SADS) of COFs determined from the global kinetic analysis of TA spectra based on the sequential kinetics with the time constant of $124 \mathrm{fs}$ and $1.25 \mathrm{ps}$. The amplitude of the first SADS was scaled for clear comparison with other SADS. The lower panel shows time-dependent population change of the SADS. The instrumental response function (dashed lines) is plotted together. c TA spectra of free-base porphyrin. $\mathbf{d}$ The upper panel shows the SADS of free-base porphyrin. The lower panel shows time-dependent population change of the SADS

units. Thus, the $\mathrm{A} \rightarrow \mathrm{B}$ transition of COFs with the time constant of $124 \mathrm{fs}$ can be attributed to the ultrafast transfer of excited charge carriers from PDI to free-base porphyrin. Meanwhile, the excitation at $523 \mathrm{~nm}$ can be resonant with both the $S_{0} \rightarrow S_{1}$ transition of PDI (peaked at $516 \mathrm{~nm}$ ) and the $\mathrm{Q}_{\mathrm{y}}$ transition of free-base porphyrin (peaked at $513 \mathrm{~nm}$ ). Although the relaxation of excited charge carriers generated from direct excitation of free-base porphyrin unit can occur, it cannot account for the large change of TA spectra induced by the $\mathrm{A} \rightarrow \mathrm{B}$ transition shown in Fig. 2b, d. In addition, the relaxation of photogenerated carriers localized in the PDI unit can occur due to the existence of defect sites that trap charge carriers. However, according to a previous TA study of perylene bisimide that is analogous to the PDI moiety ${ }^{31}$, the relaxation of excited population in the $\mathrm{S}_{1}$ state does not accompany any significant change of TA spectra in the time window up to 2 ps. By considering our fs-TA experiment on free-base porphyrin and the previous spectroscopic study of perylene bisimide ${ }^{31}$, we can confirm that the spectral change accompanying the $\mathrm{A} \rightarrow \mathrm{B}$ transition is associated with charge transfer between the building units of COFs rather than relaxation of charge carriers at local chromophores.

Coherent wavepacket features in spectroscopic data. The TA spectra of COFs shown in Fig. 2a exhibits temporal oscillations superimposed on the monotonous decay arising from the population dynamics of excited states. To extract only the temporal oscillations, we subtracted the excited-state population dynamics, which was fit by the global kinetic analysis, from the TA data. The residuals in the time domain were Fourier transformed to obtain the Fourier transform power spectra (FTPS) shown in Fig. 3a.

Although the excitation pulse is resonant only with the electronic transition of COFs, the solvent still gives non-resonant, impulsive stimulated Raman response and thus also contributes to the FTPS. To distinguish the response of neat solvent from that of COFs dispersed in the solvent, we performed a separate fs-TA measurement on neat DMF. As shown in Fig. 3a and Supplementary Fig. 11, several peaks were observed in the region of 350-1000 $\mathrm{cm}^{-1}$ over a broad range of probe wavelengths in the FTPS of both COFs and DMF, and these features are reproduced by the theoretical spectrum reconstructed from four Gaussian functions peaked at $364,405,659$, and $861 \mathrm{~cm}^{-1}$. The frequencies of these peaks observed in the FTPS of DMF are very similar to the vibrational frequencies of $354,405,667$, and $866 \mathrm{~cm}^{-1}$ observed in the Raman spectrum of DMF and thus those peaks can be assigned to the vibrations of $\mathrm{C}-\mathrm{N}$ out-of-plane bending, $\mathrm{CH}_{3}-\mathrm{N}-\mathrm{CH}_{3}$ in-plane bending, $\mathrm{O}=\mathrm{C}-\mathrm{N}$ in-plane bending, and $\mathrm{N}-\mathrm{CH}_{3}$ symmetrical stretching of $\mathrm{DMF}$, respectively ${ }^{32}$. In addition, the excitation pulse at $523 \mathrm{~nm}$ can excite the $\mathrm{Q}_{\mathrm{y}}$ transition of free-base porphyrin moiety. As shown in 
a

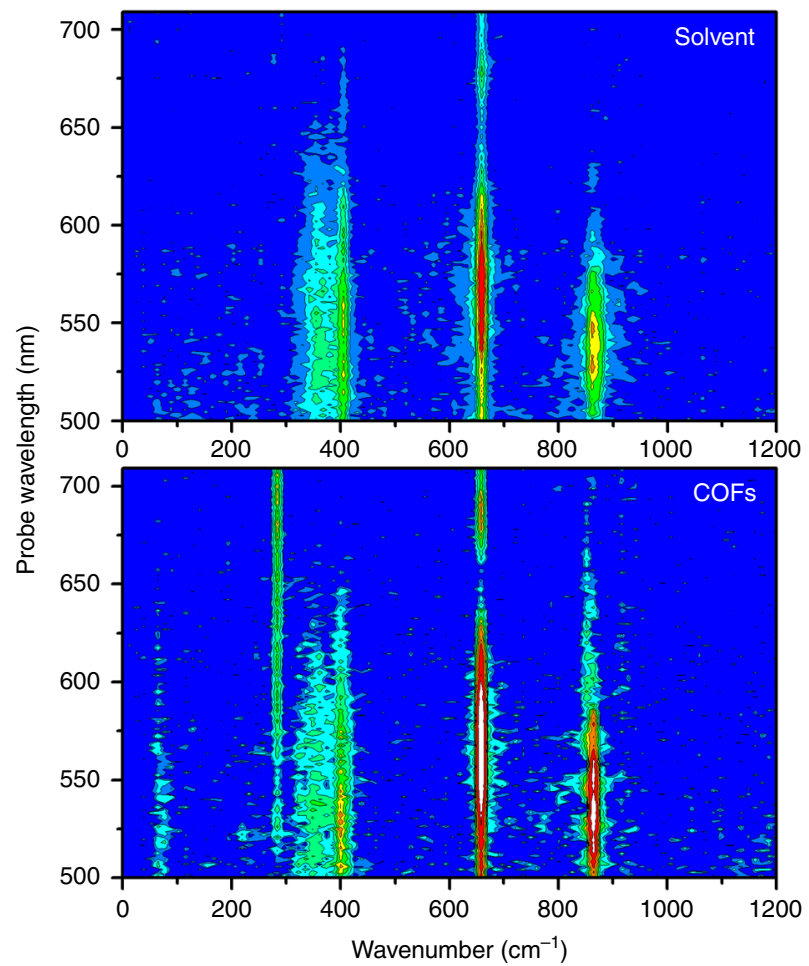

respectively. From the comparison of FTPS of COFs, neat solvent, and free-base porphyrin in the low-frequency region of $<300 \mathrm{~cm}^{-1}$, we found that the COFs exhibit two prominent peaks at 76 and $285 \mathrm{~cm}^{-1}$, which are absent in the FTPS of neat DMF and free-base porphyrin monomer.

In the 2D plot of temporal oscillations shown in Supplementary Fig. 13, we can see that the coherent oscillations persist over the entire time range of our measurement, which is in stark contrast to the electronic (or vibronic) coherences that generally undergo rapid dephasing ${ }^{18,35-37}$. As the long-lived oscillations of 76 and $285 \mathrm{~cm}^{-1}$ frequencies show nearly common phase over the entire range of probe spectrum, without any distinct nodal point (along the axis of probe wavelength) around the probe wavelength where the maximum of steady-state absorption spectrum of COFs is located, we can rule out the possibility that any of the oscillations arise from the ground-state vibrational coherence $^{38-41}$. In Fig. 3 b, we compared the slices of wavelengthresolved FTPS at selected oscillation frequencies with the SADS of COFs. The FTPS slice at $76 \mathrm{~cm}^{-1}$ has a similar spectral shape as the SE signal of the first SADS, whereas the FTPS slice at $285 \mathrm{~cm}^{-1}$ has non-zero amplitude in the entire spectral range of the second SADS. We note that the first SADS in the $490-625 \mathrm{~nm}$ region is dominated by the SE of PDI units. Based on this result, we infer that the long-lived oscillations of 76 and $285 \mathrm{~cm}^{-1}$ frequencies can be assigned to molecular vibrations of excitedstate COFs, specifically the former is related with the PDI units.

b

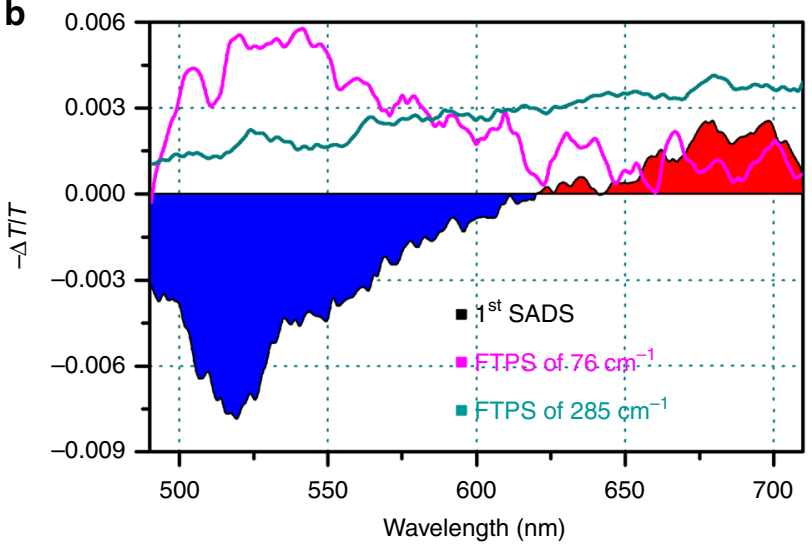

Fig. 3 Wavelength-resolved Fourier transform power spectra. a Wavelength-resolved Fourier transform power spectra (FTPS) of neat DMF (top) and COFs dispersed in DMF (bottom) with the probe spectral window of $500-710 \mathrm{~nm}$. The Fourier power spectra of COFs have peaks at 76 and $285 \mathrm{~cm}^{-1}$, which are absent in the FTPS of pure solvent. b Comparison of SADS (black) with the slices of wavelength-resolved FTPS at selected oscillation frequencies. In the SADS, the blue- and the redshaded area indicate negative and positive amplitude of the TA signal, respectively. The amplitude of the $76 \mathrm{~cm}^{-1}$ oscillation is dominant in the spectral range of ground-state bleaching and stimulated emission of the first SADS, whereas the $285 \mathrm{~cm}^{-1}$ mode is manifested in the entire spectral range of probe pulse

Supplementary Fig. 12, the FTPS from the separate fs-TA measurement of free-base porphyrin shows several major peaks, of which vibrational frequencies of 85 and $321 \mathrm{~cm}^{-1}$ are distinctly different from the frequencies of $354,405,667$, and $866 \mathrm{~cm}^{-1}$ observed in the neat DMF. Based on the previously reported resonance Raman and theoretical studies ${ }^{33,34}$, the two peaks at 85 and $321 \mathrm{~cm}^{-1}$ can be assigned to the torsional motion of side phenyl rings and the in-plane stretching of porphyrin,
Ab initio non-adiabatic molecular dynamics simulation. NAMD simulations based on time-dependent Kohn-Sham DFT $(\mathrm{KS}-\mathrm{DFT})^{42,43}$ was performed to investigate the ultrafast dynamics of charge carriers observed in the fs-TA measurement. The structure of COFs was modeled by using a periodic unit cell of monolayer (Supplementary Fig. 14) and computational details are described in Methods. To circumvent the problem of underestimating the optical bandgap for the KS energy states (Supplementary Fig. 15), scissor operations were applied to the virtual KS orbital levels (Supplementary Figs. 16 and 17) in order to reproduce the major peaks in the experimental absorption spectrum in the 500-700 $\mathrm{nm}$ region. The KS levels were labeled using the highest occupied molecular orbital (HOMO) $-n$ and lowest unoccupied mmolecular orbital (LUMO) $+m$ notation, which denote the occupied and virtual KS states, respectively, of the simulated COF system, where the larger $n$ (or $m$ ) means the state located farther away from the Fermi level. Due to the quasi fourfold rotational symmetry with respect to the center of freebase porphyrin unit in the $2 \mathrm{D}$ COFs, the nearly degenerate KS states were allowed and distinguished using the prime notation; e.g., $\mathrm{HOMO}-n^{\prime}$ or LUMO $+m^{\prime}$. The low-energy singly excited states, which were chosen as the active space of our surface-hopping $(\mathrm{SH})$ simulations, are labeled as $\mathrm{S}_{\mathrm{a}}$ to $\mathrm{S}_{\mathrm{x}}$ (note that the excited states are labeled in terms of LUMO energy in ascending order); the locations of an electron and a hole in each excited state are summarized in Supplementary Table 1.

From the NA-MD simulation, we found that the photoexcitation at $523 \mathrm{~nm}$ employed in the fs-TA measurement induces the following: (1) an electronic transition from HOMO-3 to LUMO, which corresponds to the $S_{0}$-to- $S_{b}$ transition involving the KS orbitals mainly located along the vertical axis of COFs, and (2) an electronic transition from HOMO-3' to $\mathrm{LUMO}^{\prime}$, which corresponds to the $\mathrm{S}_{0}$-to- $\mathrm{S}_{\mathrm{i}}$ transition involving the KS orbitals mainly located along the horizontal axis of COFs. For clarity, the directions of vertical and horizontal axes in the structure of COFs are indicated in Fig. 1a. We note that all of the orbitals involved in these transitions are localized at the PDI unit of COFs. The NA-MD simulation results show that the $\mathrm{S}_{\mathrm{b}}$ state 

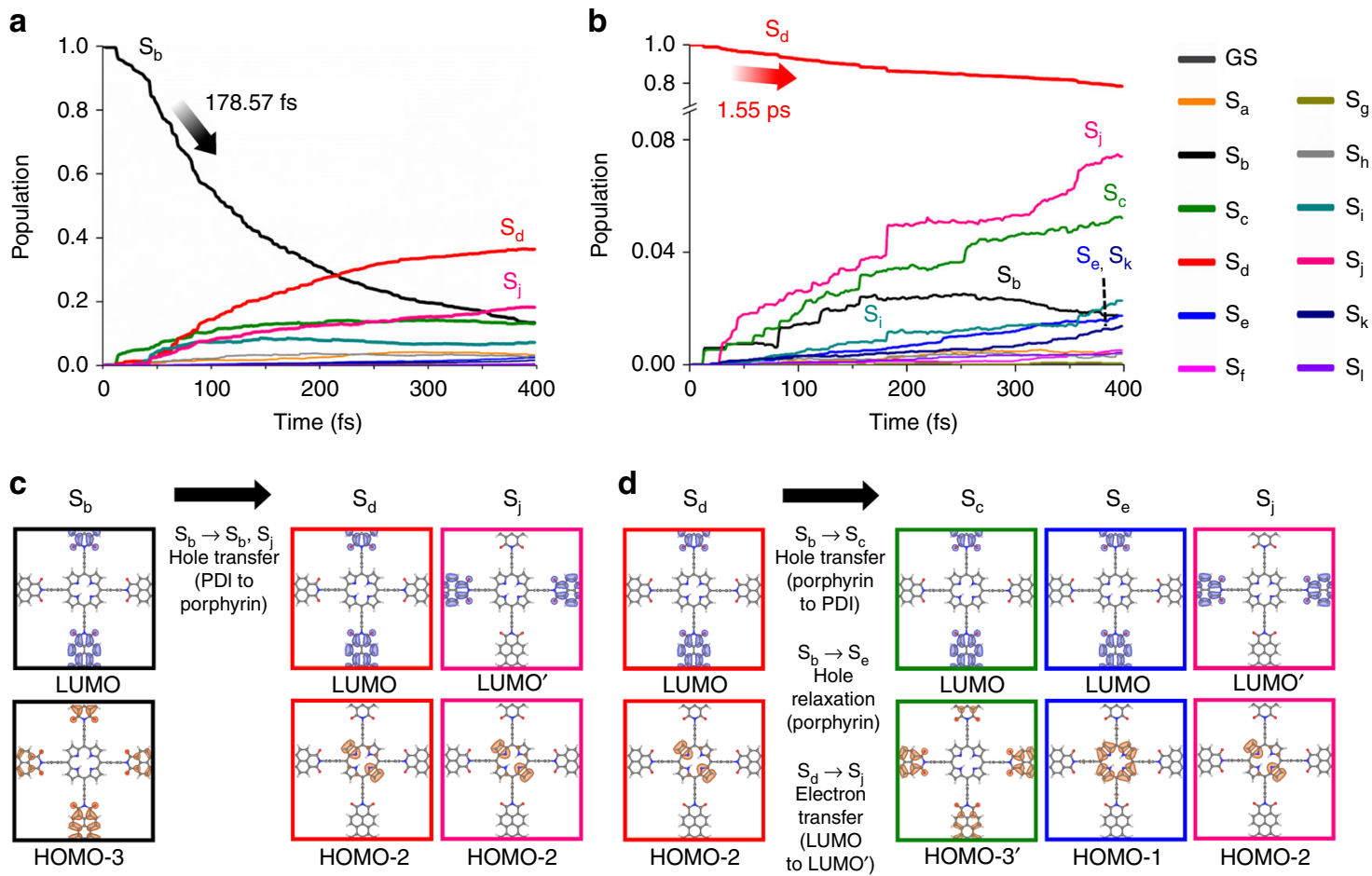

Fig. $4 \mathrm{Ab}$ initio non-adiabatic molecular dynamics simulations. a Time evolution of electronic populations starting from the initially excited $\mathrm{S}_{\mathrm{b}}$ state, where the charge carriers are localized in the PDI moieties. $\mathbf{b}$ Time evolution of electronic populations starting from the hole-transferred state $\left(\mathrm{S}_{\mathrm{d}}\right)$, describing the dynamics of charge carriers after PDI-to-porphyrin hole transfer. c Evolution of electron-hole distribution starting from the initially excited $\mathrm{S}_{\mathrm{b}}$ state, visualized with the Kohn-Sham (KS) orbitals of COFs. In each of HOMO and LUMO, the densities of holes (orange) and electrons (blue) are depicted. All possible electron-hole pairs in the active space are listed in Supplementary Table 2. d Evolution of electron-hole distribution starting from the hole-transferred state $S_{d}$. In each of HOMO and LUMO, the densities of holes (orange) and electrons (blue) are depicted

decays with a time constant of $179 \mathrm{fs}$ (Fig. $4 \mathrm{a}$ and Supplementary Table 2) and, as a result, the populations of $S_{d}$ and $S_{j}$ states increase predominantly. The $S_{d}$ and $S_{j}$ states have the chargetransfer character with the hole located at porphyrin (HOMO-2) and the electron located at PDI (LUMO in the vertical direction and $\mathrm{LUMO}^{\prime}$ in the horizontal direction, respectively). Therefore, the transition from $S_{b}$ to $S_{d}$ or $S_{j}$ involves hole transfer from PDI to porphyrin. The $S_{i}$ excited state, which consists of KS orbitals mainly located along the horizontal axis, also relaxes to $S_{d}$ and $S_{j}$ with the time constant of $173 \mathrm{fs}$, which is a time scale comparable with that of relaxation dynamics of $S_{b}$ state (179 fs time constant) located along the vertical axis, as shown in Supplementary Fig. 18 and Supplementary Table 2.

From the additional set of NA-MD simulations, the relaxation dynamics of the $S_{d}$ state was also investigated. The $S_{d}$ state decays slowly with a time constant of $1.55 \mathrm{ps}$ (Fig. 4b). Concomitantly, the populations of $S_{b}, S_{c}, S_{e}, S_{i}, S_{j}$, and $S_{k}$ states increase noticeably. The $S_{b}, S_{c}$, and $S_{i}$ states have both the hole (HOMO-3 and $\mathrm{HOMO}-3^{\prime}$ ) and the electron (LUMO and LUMO') located at the PDI moieties, indicating the back transfer of holes from porphyrin (HOMO-2) to PDI (HOMO-3 and HOMO-3') by the transition from $S_{d}$ to $S_{b}, S_{c}$, and $S_{i}$. The $S_{e}$ and $S_{k}$ states have the hole at porphyrin (HOMO-1) and the electron at PDI (LUMO and LUMO'), indicating the relaxation of holes from HOMO-2 to HOMO-1 (at porphyrin) by the transition from $S_{d}$ to $S_{e}$ and $S_{k}$. In the case of the $S_{j}$ state, the electron is located at the horizontal PDI moieties and the hole is at the porphyrin units, indicating the transfer of electrons from vertical PDI (LUMO) to horizontal PDI (LUMO') by the transition from $S_{d}$ to $S_{j}$.

In addition to the charge carrier dynamics of COFs, vibrational normal modes of the COF monolayer were investigated in order to examine the structural motions associated with the charge carrier dynamics. For readers with interest, all calculated vibrational motions are provided using the animated GIFs in the Supplementary Data 1-13 To investigate the vibrational modes related with the coherent oscillations observed in the fs-TA data, the calculated vibrational modes with the frequencies near 76 and $285 \mathrm{~cm}^{-1}$ are summarized in Supplementary Table 3, and the histogram of calculated vibrational modes with frequencies less than $300 \mathrm{~cm}^{-1}$ is shown in Supplementary Fig. 19.

\section{Discussion}

The combined results of the fs-TA measurement and the NA-MD simulation provide comprehensive information on the charge carrier dynamics of COFs with the D-A conjugation. In the fs-TA measurement, it was observed that $\mathrm{SE}$ is quenched with $124 \mathrm{fs}$ time constant by the $\mathrm{A} \rightarrow \mathrm{B}$ transition, which is assigned to charge transfer from PDI to porphyrin. In order to examine the excitation condition of fs-TA experiment, the theoretical linear absorption spectrum of COFs was calculated from the KS-DFT levels in conjunction with the ground-state MD simulation. As shown in Supplementary Fig. 17, the excitation at $523 \mathrm{~nm}$ can be resonant with both the HOMO-3 $\rightarrow$ LUMO transition localized in the PDI moiety (peaked at $514 \mathrm{~nm}$ ) and the HOMO- $1 \rightarrow$ $\mathrm{LUMO}+1^{\prime}$ transition localized in the porphyrin moiety (peaked at $513 \mathrm{~nm}$ ). As the transition probability of HOMO-3 $\rightarrow$ LUMO is twice larger than that of HOMO- $\rightarrow \mathrm{LUMO}+1^{\prime}$, the photoexcitation at $523 \mathrm{~nm}$ is likely to dominantly excite the optical transition localized in the PDI moiety. According to the NA-MD simulation on the charge carrier dynamics of COFs, the population decay of initially excited $S_{b}$ state, which corresponds to the transition of HOMO-3 $\rightarrow$ LUMO, leads to concurrent growth of 
the populations of $S_{d}$ and $S_{j}$ states with the time constant of $\sim 180$ fs, matching the time scale of the ultrafast quenching of $\mathrm{SE}$ observed in the fs-TA data. As $S_{d}$ and $S_{j}$ states have holes at the porphyrin units and electrons at the PDI units as shown above, the transition from $S_{b}$ to $S_{d}$ or $S_{j}$ induces hole transfer from PDI to porphyrin, resulting in the formation of a polaron pair in the 2D lattice of D-A COFs. Thus, the NA-MD simulation not only confirms the assignment of $\mathrm{A} \rightarrow \mathrm{B}$ transition but also reveals that it is the hole carrier that is transferred from PDI to porphyrin.

The ultrafast charge transfer identified in the present work may originate from the quasi-degeneracy of HOMO-3 and HOMO-2 orbitals, which constitute $S_{b}$ and $S_{d} / S_{j}$ states, respectively, and are located along the vertical axis of COFs. The similar energies of the two orbitals are expected to increase the rate of hole transfer in the vertical direction. Such quasi-degeneracy is also observed between HOMO-3' and HOMO-2 orbitals located along the horizontal axis of COFs, suggesting ultrafast hole transfer (occurring with the transition from $S_{i}$ to $S_{d}$ and $S_{j}$ ) in the horizontal direction as well.

In addition to the ultrafast dynamics of hole transfer, the kinetic component of 1.25 ps time constant is identified in the TA spectra and assigned to the $\mathrm{B} \rightarrow \mathrm{C}$ transition. According to the NA-MD simulation, the relaxation of $S_{d}$ occurs with 1.55 ps time constant, which matches the time scale of the $\mathrm{B} \rightarrow \mathrm{C}$ transition. Therefore, this kinetic component can be assigned to the relaxation of the polaron pair states, i.e., (1) porphyrinto-PDI back transfer of hole carriers (to $\mathrm{S}_{\mathrm{b}} / \mathrm{S}_{\mathrm{c}} / \mathrm{S}_{\mathrm{i}}$ ), (2) hole relaxation at porphyrin (to $\mathrm{S}_{\mathrm{e}} / \mathrm{S}_{\mathrm{k}}$ ), and (3) electron transfer from vertical to horizontal PDI (to $\left.S_{j}\right)^{8,22}$. We note that a the current NA-MD simulation of COFs was implemented for single-layered COFs, only intra-layer charge transfer, not inter-layer charge transport, was considered. Meanwhile, in a previous TA study on D-A COFs composed of zinc phthalocyanine and naphthalene diimide (i.e., ZnPc-NDI COFs) ${ }^{22}$, the earliest kinetic component with 1.8 ps time constant was observed and assigned to photoinduced electron transfer from $\mathrm{ZnPc}$ to NDI unit and subsequent inter-layer charge delocalization along separate donor and acceptor arrays perpendicular to stacked 2D layers. Therefore, considering the similarity of the time scales of the kinetic components obtained from the two TA studies (1.25 ps vs. $1.8 \mathrm{ps}$ ), the $1.25 \mathrm{ps}$ component may be attributed to inter-layer charge transport.

Although the pore size of COFs determined from the nitrogen isotherm shown in Supplementary Fig. 5 is close to that of theoretically modeled structure shown in Supplementary Fig. 2 (see Supplementary Note 3), we cannot completely rule out the presence of defects such as mislocation of building units in a $2 \mathrm{D}$ layer or less-ordered stacking between the 2D layers. As the defects in the 2D layer may retard or block the excitons initially formed in the PDI moieties from being split into charge carriers (i.e., electrons and holes) through charge transfer between PDI and porphyrin, the spectroscopically measured rates of charge transfer can be regarded as the lower limit for the defect-free material. Considering the prominent change of TA spectra associated with the ultrafast charge transfer between the building units in an individual 2D layer, the observed dynamics is highly unlikely to originate from the defects in a 2D layer. The analyses of TEM images and PXRD pattern show that the synthesized COFs adopts the staggered structure along the $c$-axis with slipping along $a$-axis and/or $b$-axis, as shown in Supplementary Fig. 2b. Such staggered inter-layer structure would result in inefficient charge migration between the stacked 2D layers. Moreover, considering that the TA measurement was conducted for the COF sample dispersed in solution phase, it is possible that the stacked layers in the solid phase can be separated into much thinner stacks or even single layers. All these considerations lead us to conclude that the electrons and holes generated from the photoinduced charge transfer are likely to undergo charge recombination only within individual $2 \mathrm{D}$ layers.

The coherent oscillations of $76 \mathrm{~cm}^{-1}$ and $285 \mathrm{~cm}^{-1}$ frequencies observed in the fs-TA data reflect lattice vibrations coupled with the electronic transition of COFs. The detailed motions of those lattice vibrations can be examined with vibrational normal mode analysis of COFs. As the calculated lattice vibrations in the reticular structure of COFs show complex patterns of motions, we describe those motions in terms of vibrations of constituent units of COFs. The vibrational modes of $74.02 \mathrm{~cm}^{-1}$ and $77.12 \mathrm{~cm}^{-1}$ have the closest frequencies to the $76 \mathrm{~cm}^{-1}$ oscillation identified in the fs-TA measurement and these modes correspond to collective motions including (i) saddling motion of PDI moiety, (ii) in-plane and out-of-plane motions of free-base porphyrin moiety, and (iii) torsional motion of benzene ring that covalently links PDI and free-base porphyrin. The vibrational modes of $282.08 \mathrm{~cm}^{-1}$ and $286.26 \mathrm{~cm}^{-1}$ frequencies have the closest frequencies to the $285 \mathrm{~cm}^{-1}$ oscillation identified in the fs-TA data, and they contain (i) in-plane distortion of PDI moiety without any rotating motion of benzene linker and (ii) in-plane and out-of-plane motions of free-base porphyrin moiety. According to the vibrational normal mode analysis, the distinct difference between the $76 \mathrm{~cm}^{-1}$ mode and the $285 \mathrm{~cm}^{-1}$ mode is the change of the dihedral angle between the benzene ring and the layer plane of PDI and porphyrin; this angle changes in the $76 \mathrm{~cm}^{-1}$ mode, whereas it is almost kept constant in the $285 \mathrm{~cm}^{-1}$ mode. The torsional motion of the benzene linker in the $76 \mathrm{~cm}^{-1}$ mode can enhance its $\pi$-interaction with the PDI and porphyrin by planarization of PDI-benzene-porphyrin units and resultantly facilitates charge transfer between PDI and porphyrin. Therefore, the coherent oscillation of $76 \mathrm{~cm}^{-1}$ frequency observed in the fs-TA data is evidence of the intra-layer charge transfer mediated by the torsional motions of intervening $\pi$-bridge units. Such an ultrafast intramolecular charge transfer driven by the torsional motion of $\pi$-bridge has been also observed in various donor-bridgeacceptor copolymers ${ }^{44,45}$. Based on the population dynamics and the vibrational normal modes of COFs obtained from the quantum mechanical simulation, we conclude that the ultrafast dynamics observed in the fs-TA signal of 2D COFs originate from the ultrafast formation of polaron pair through hole migration between the constituent donor and acceptor units of COFs and such hole transfer is mediated by motions of lattice phonons. We summarize the results of the fs-TA measurement and the NA-MD simulations in Fig. 5, representing the phonon-assisted charge transfer in the $2 \mathrm{D}$ D-A COFs.

In this work, by combining fs-TA spectroscopy and NA-MD simulation, we directly observed ultrafast dynamics of phononassisted, PDI-to-porphyrin hole transfer in 2D COFs consisting of covalently linked electron donor and acceptor units. The key findings of this work can be utilized for the design of COF-based light-harvesting module applicable to high-performance optoelectronic devices. For example, the 2D D-A COFs can be combined with COFs consisting of only free-base porphyrins. The free-base porphyrin COFs in the form of well-stacked 2D layers exhibit high hole mobility through a periodically ordered macrocycle channel ${ }^{8,21}$. Such hybrid COF architecture will be able to achieve efficient charge separation and charge transport of hole carriers, which are desirable for highly efficient photovoltaic devices.

\section{Methods}

Synthesis of COFs. For the synthesis of COFs, perylene-3,4,9,10-tetracarboxylic dianhydride and 5,10,15,20-tetrakis(4-aminophenyl)-21H,23H-porphyrin were prepared separately and then linked together by the polymerization process. In a 


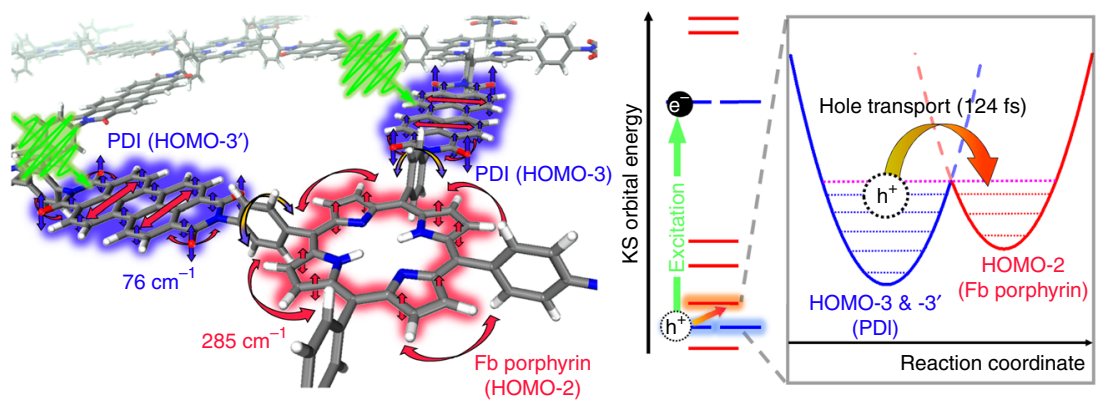

Fig. 5 Schematic of charge carrier dynamics in photoinduced COFs. Upon photoexcitation of COFs, ultrafast hole transfer through the splitting of excitons occurs from PDI to free-base porphyrin (Fb porphyrin) unit with the time constant of $124 \mathrm{fs}$. According to the NA-MD simulation, the quasi-degenerate electronic states of HOMO-3 (or - $3^{\prime}$ ) and HOMO-2 facilitate such ultrafast transfer of charge carriers. The coherent oscillations with the frequencies of $76 \mathrm{~cm}^{-1}$ and $285 \mathrm{~cm}^{-1}$ superimposed on the decay of TA spectra are associated with coherent lattice phonons of COFs. Especially, the change in the dihedral angle of benzene linker (yellow arrow) can be related with the motions that enhance $\pi$-interaction between PDI and porphyrin units, resulting in the ultrafast transfer of charge carriers

$500 \mathrm{ml}$ two-neck round-bottle flask, perylene-3,4,9,10-tetracarboxylic dianhydride $(4.00 \mathrm{~g}, 15 \mathrm{mmol})$ was dissolved in anhydrous pyridine $(90 \mathrm{~mL})$. To this solution, a solution of 5,10,15,20-tetrakis(4-aminophenyl)-21H,23H-porphyrin (3.23 g, 25 $\mathrm{mmol})$ in pyridine $(40 \mathrm{~mL})$ was added drop wise. After overnight stirring at $130^{\circ} \mathrm{C}$ in argon atmosphere, the reaction mixture was cooled down to room temperature. The reddish-purple precipitate was filtered and washed with copious amount of distilled water, methanol, and chloroform. After drying in the oven at $110^{\circ} \mathrm{C}$, pale purple solid product was obtained. The details for the synthesis and the characterization of COFs are described in the Supplementary Note 1-3.

fs-TA spectroscopy. TA spectra were measured with fs laser pulses using a visible pump-broadband probe scheme. The output pulses at $800 \mathrm{~nm}$ wavelength from a Ti:sapphire amplified laser (Coherent Legend Elite) were split into pump and probe beams. On the pump arm, the $800 \mathrm{~nm}$ laser pulses were converted into the pump pulses of $523 \mathrm{~nm}$ wavelength and $20 \mathrm{~nm}$ bandwidth using a homebuilt, all-reflective-optic noncollinear optical parametric amplifier. The pump pulses were sent through a pair of Brewster-cut fused-silica prisms to precompensate for the dispersion obtained from transmissive optics and compressed to near-transform-limited pulses at the sample position. On the probe arm, the $800 \mathrm{~nm}$ laser pulses were sent into a $c$-cut sapphire window of $3 \mathrm{~mm}$ thickness and converted into white light continuum spanning from visible to near-infrared wavelengths by self-phase modulation. The visible portion $(460-720 \mathrm{~nm})$ of the white light continuum was used as broadband probe pulses without further compensation of the dispersion. The probe pulses were time-delayed with respect to the pump pulses using a motorized translation stage (Newport, M-ILS150HA). By recording "pump-on" and "pump-off" probe spectra, the differential transmission $(\Delta T / T)$ spectrum was obtained as a function of time. The spectra of transient signal and the reference were detected by a spectrometer (Andor, SR303i) equipped with a Si CCD (Andor, DU420A). In all the measurements, the polarization of the pump pulses was set to be at a magic angle $\left(54.7^{\circ}\right)$ relative to the probe polarization in order to prevent polarization-dependent signals. For the TA measurement, 2D COFs were suspended in DMF, which causes the exfoliation of the $2 \mathrm{D}$ COF layers from the bulk sample through sonication process. This exfoliation procedure improves solubility of COFs that is well suited for the TA spectroscopic measurement ${ }^{22,46}$. The pulse energy of the pump pulses was varied from 63 to $125 \mathrm{~nJ}$ to check the excitation energy dependence of TA signal. As the decay profiles of TA data were not dependent on the fluence of pump pulse shown in Supplementary Fig. 20, we did not consider the contribution of exciton-exciton annihilation in the time window of our TA measurement. For the broadband TA measurement, the excitation energy was set to $125 \mathrm{~nJ}$ at the sample position in order to achieve a high signal-to-noise ratio. The broadband TA data were measured at various time delays in the time range from -440 to $2728 \mathrm{fs}$ with a time step of $8 \mathrm{fs}$. To avoid unwanted scatterings of the excitation beam mixed into the TA signal, we cautiously positioned the sample cell in order to reduce any unwanted scattering off the surfaces of the cell. From impulsive stimulated Raman scattering measurement of tetrachloromethane $\left(\mathrm{CCl}_{4}\right)$, the instrumental response function of the TA measurement was determined to be $68 \mathrm{fs}$ from the FWHM of cross-correlation function of the pump and the probe pulses measured at the sample position. The solvent signals were measured with neat DMF in the identical quartz cell as used in the measurement of the COFs sample dissolved in DMF.

Quantum mechanical simulation. We performed NA-MD simulations as implemented in the PYXAID code ${ }^{42,43}$. This method employs the classical path approximation (CPA $)^{47,48}$-assuming the evolution of nuclear dynamics being independent of the electrons, which allows the practical investigation of the realtime relaxation dynamics of the photoexcited systems with periodic boundary conditions by means of the SH with KS-DFT states. We note that the validity of
CPA has been tested against a number of systems, in which electronic excitation barely affects the nuclear dynamics according to previous studies ${ }^{49-52}$. In this regard, the charge-transfer dynamics, accompanied by the structural motion, can be properly explained by the NA-MD simulation based on the PYXAID code.

Using Quantum ESPRESSO program ${ }^{53}$, we first optimized a unit cell of COFs (Supplementary Fig. 14) consisting of the two PDIs and one free-base porphyrin subunit, and then performed a ground-state MD simulation for $500 \mathrm{fs}$. We employed the Perdew-Burke-Ernzerhof (PBE) exchange-correlation $(x c)$ functional ${ }^{54}$ with a plane-wave cutoff energy of $35 \mathrm{Ry}$ and only the gamma point is sampled in the reciprocal space. After applying scissor operations to the virtual states to reproduce the experimental absorption energy (Supplementary Fig. 17), the NA-MD simulations were then performed using the PYXAID code. Active space for the SH dynamics was chosen as the range from HOMO- 4 to LUMO $+1^{\prime}$ after a thorough convergence check upon the increase of active space size (Supplementary Table 4). All possible single excitation states in the active space are labeled from $S_{a}$ to $S_{x}$ as listed in Supplementary Table 1.

To obtain statistically meaningful population dynamics of excited states, five $400 \mathrm{fs}$ NA-MD trajectories were obtained and averaged. The time constant, $\tau$, for the decay of population was determined by fitting the temporal change of excitedstate population with an exponential-decay function (1) as follows:

$$
\mathrm{P}(t)=\exp \left(-\frac{t}{\tau}\right)
$$

We also found that the overcoherence problem of fewest-switches $\mathrm{SH}^{49,55}$ should be corrected to obtain a reasonable time constant (Supplementary Fig. 21) and thus we employed decoherence-induced $\mathrm{SH}^{56}$ scheme. For the calculations of PXRD patterns and vibrational modes, we used Vienna ab initio simulation package ${ }^{57,58}$ with the choice of same PBE xc-functional and the plane-wave cutoff energy of 450 $\mathrm{eV}$.

\section{Data availability}

The data that support the findings of this study are available from the corresponding author upon reasonable request.

Received: 22 October 2018 Accepted: 4 April 2019

Published online: 23 April 2019

\section{References}

1. Côté, A. P. et al. Porous, crystalline, covalent organic frameworks. Science 310, 1166-1170 (2005)

2. Waller, P. J., Gándara, F. \& Yaghi, O. M. Chemistry of covalent organic frameworks. Acc. Chem. Res. 48, 3053-3063 (2015).

3. Feng, X., Ding, X. S. \& Jiang, D. L. Covalent organic frameworks. Chem. Soc. Rev. 41, 6010-6022 (2012).

4. Ding, S. Y. \& Wang, W. Covalent organic frameworks (COFs): from design to applications. Chem. Soc. Rev. 42, 548-568 (2013).

5. Colson, J. W. \& Dichtel, W. R. Rationally synthesized two-dimensional polymers. Nat. Chem. 5, 453-465 (2013).

6. Farha, O. K. et al. Synthesis, properties, and gas separation studies of a robust diimide-based microporous organic polymer. Chem. Mater. 21, 3033-3035 (2009).

7. Calik, M. et al. Extraction of photogenerated electrons and holes from a covalent organic framework integrated heterojunction. J. Am. Chem. Soc. 136, 17802-17807 (2014). 
8. Feng, X. et al. High-rate charge-carrier transport in porphyrin covalent organic frameworks: switching from hole to electron to ambipolar conduction. Angew. Chem. Int. Ed. 51, 2618-2622 (2012).

9. Yadav, R. K., Kumar, A., Park, N. J., Kong, K. J. \& Baeg, J. O. A highly efficient covalent organic framework film photocatalyst for selective solar fuel production from CO2. J. Mater. Chem. A 4, 9413-9418 (2016).

10. Medina, D. D. et al. Oriented thin films of a benzodithiophene covalent organic framework. ACS Nano. 8, 4042-4052 (2014).

11. Clarke, T. M. \& Durrant, J. R. Charge photogeneration in organic solar cells. Chem. Rev. 110, 6736-6767 (2010).

12. Rolczynski, B. S. et al. Ultrafast intramolecular exciton splitting dynamics in isolated low-band-gap polymers and their implication in photovoltaic materials design. J. Am. Chem. Soc. 134, 4142-4152 (2012).

13. Grancini, G. et al. Hot exciton dissociation in polymer solar cells. Nat. Mater. 12, 29-33 (2013).

14. Tautz, R. et al. Structural correlations in the generation of polaron pairs in low-bandgap polymers for photovoltaics. Nat. Commun. 3, 970 (2012).

15. Paraecattil, A. A., Beaupre, S., Leclerc, M., Moser, J. E. \& Banerji, N. Intensity Dependent femtosecond dynamics in a PBDTTPD-based solar cell Mmaterial. J. Phys. Chem. Lett. 3, 2952-2958 (2012).

16. Bakulin, A. A. et al. The role of driving energy and delocalized states for charge separation in organic semiconductors. Science 335, 1340-1344 (2012).

17. Ziolek, M. et al. Femtosecond to millisecond studies of electron transfer processes in a donor-(pi-spacer)-acceptor series of organic dyes for solar cells interacting with titania nanoparticles and ordered nanotube array films. Phys. Chem. Chem. Phys. 14, 2816-2831 (2012).

18. Jun, S. et al. Coherent oscillations in chlorosome elucidated by twodimensional electronic spectroscopy. J. Phys. Chem. Lett. 5, 1386-1392 (2014).

19. Kleima, F. J. et al. Forster excitation energy transfer in peridinin-chlorophylla-protein. Biophys. J. 78, 344-353 (2000)

20. Dahlberg, P. D., Fidler, A. F., Caram, J. R., Long, P. D. \& Engel, G. S. Energy transfer observed in live cells using two-dimensional electronic spectroscopy. J. Phys. Chem. Lett. 4, 3636-3640 (2013).

21. Jin, S. B. et al. Creation of superheterojunction polymers via direct polycondensation: segregated and bicontinuous donor-acceptor pi-columnar arrays in covalent organic frameworks for long-lived charge separation. J. Am. Chem. Soc. 137, 7817-7827 (2015).

22. Jin, S. B. et al. Charge dynamics in a donor-acceptor covalent organic framework with periodically ordered bicontinuous heterojunctions. Angew. Chem. Int. Ed. 52, 2017-2021 (2013).

23. Baskin, J. S., Yu, H. Z. \& Zewail, A. H. Ultrafast dynamics of Porphyrins in the condensed phase: I. Free base tetraphenylporphyrin. J. Phys. Chem. A 106, 9837-9844 (2002).

24. Rury, A. S. \& Sension, R. J. Broadband ultrafast transient absorption of iron (III) tetraphenylporphyrin chloride in the condensed phase. Chem. Phys. 422, 220-228 (2013).

25. Kullmann, M. et al. Ultrafast exciton dynamics after Soret- or Q-band excitation of a directly beta, beta '-linked bisporphyrin. Phys. Chem. Chem. Phys. 14, 8038-8050 (2012).

26. Long, S. R. et al. Energy transfer and spectroscopic characterization of a perylenetetracarboxylic diimide (PDI) hexamer. Phys. Chem. Chem. Phys. 17, 18567-18576 (2015).

27. Lim, J. M. et al. Exciton delocalization and dynamics in helical pi-stacks of self-assembled perylene bisimides. Chem. Sci. 4, 388-397 (2013).

28. Bruggemann, B., Persson, P., Meyer, H. D. \& Maya, V. Frequency dispersed transient absorption spectra of dissolved perylene: a case study using the density matrix version of the MCTDH method. Chem. Phys. 347, 152-165 (2008).

29. Kim, S. Y. \& Joo, T. Coherent nuclear wave packets in Q states by ultrafast internal conversions in free base tetraphenylporphyrin. J. Phys. Chem. Lett. 6, 2993-2998 (2015).

30. Akimoto, S., Yamazaki, T., Yamazaki, I. \& Osuka, A. Excitation relaxation of zinc and free-base porphyrin probed by femtosecond fluorescence spectroscopy. Chem. Phys. Lett. 309, 177-182 (1999).

31. Son, M., Park, K. H., Yoon, M. C., Kim, P. \& Kim, D. Excited-state vibrational coherence in perylene bisimide probed by femtosecond broadband pumpprobe spectroscopy. J. Phys. Chem. A 119, 6275-6282 (2015).

32. Chalapathi, V. V. \& Ramiah, K. V. Normal vibrations of N, Ndimethylformamide and N, N-dimethylacetamide. Proc. Indian Acad. Sci. A 68, 109-122 (1968)

33. Saini, G. S. S. Resonance Raman study of free-base tetraphenylporphine and its dication. Spectrochim. Acta A 64, 981-986 (2006).

34. Minaev, B., Wang, Y. H., Wang, C. K., Luo, Y. \& Agren, H. Density functional theory study of vibronic structure of the first absorption $\mathrm{Q}(\mathrm{x})$ band in freebase porphin. Spectrochim. Acta A 65, 308-323 (2006).

35. Cheng, Y. C. \& Fleming, G. R. Coherence quantum beats in two-dimensional electronic spectroscopy. J. Phys. Chem. A 112, 4254-4260 (2008).

36. Turner, D. B., Wilk, K. E., Curmi, P. M. G. \& Scholes, G. D. Comparison of electronic and vibrational coherence measured by two-dimensional electronic spectroscopy. J. Phys. Chem. Lett. 2, 1904-1911 (2011)
37. Halpin, A. et al. Two-dimensional spectroscopy of a molecular dimer unveils the effects of vibronic coupling on exciton coherences. Nat. Chem. 6, 196-201 (2014)

38. Dhar, L., Rogers, J. A. \& Nelson, K. A. Time-resolved vibrational spectroscopy in the impulsive limit. Chem. Rev. 94, 157-193 (1994).

39. Pollard, W. T. et al. Theory of dynamic absorption-spectroscopy of nonstationary states .4. Application to 12-Fs resonant impulsive Ramanspectroscopy of bacteriorhodopsin. J. Phys. Chem. 96, 6147-6158 (1992).

40. Dean, J. C. et al. Broadband transient absorption and two-dimensional electronic spectroscopy of methylene blue. J. Phys. Chem. A 119, 9098-9108 (2015).

41. Ikuta, M., Yuasa, Y., Kimura, T., Matsuda, H. \& Kobayashi, T. Phase analysis of vibrational wave packets in the ground and excited states in polydiacetylene. Phys. Rev. B 70, 214301 (2004).

42. Akimov, A. V. \& Prezhdo, O. V. The PYXAID program for non-adiabatic molecular dynamics in condensed matter systems. J. Chem. Theory Comput. 9 , 4959-4972 (2013)

43. Akimov, A. V. \& Prezhdo, O. V. Advanced capabilities of the PYXAID program: integration schemes, decoherence: effects, multiexcitonic states, and field-matter interaction. J. Chem. Theory Comput. 10, 789-804 (2014).

44. Roy, P. et al. Ultrafast bridge planarization in donor-pi-acceptor copolymers drives intramolecular charge transfer. Nat. Commun. 8, 1716 (2017).

45. Hwang, I., Beaupre, S., Leclerc, M. \& Scholes, G. D. Ultrafast relaxation of charge-transfer excitons in low-bandgap conjugated copolymers. Chem. Sci. 3, 2270-2277 (2012)

46. Berlanga, I. et al. Delamination of layered covalent organic frameworks. Small 7, 1207-1211 (2011).

47. Fischer, S. A., Habenicht, B. F., Madrid, A. B., Duncan, W. R. \& Prezhdo, O. V. Regarding the validity of the time-dependent Kohn-Sham approach for electron-nuclear dynamics via trajectory surface hopping. J. Chem. Phys. 134, 024102 (2011)

48. Craig, C. F., Duncan, W. R. \& Prezhdo, O. V. Trajectory surface hopping in the time-dependent Kohn-Sham approach for electron-nuclear dynamics. Phys. Rev. Lett. 95, 163001 (2005).

49. Wei, Y., Zhou, Z. \& Long, R. Defects slow down nonradiative electron-hole recombination in TiS3 nanoribbons: a time-domain ab initio study. J. Phys. Chem. Lett. 8, 4522-4529 (2017).

50. Senanayake, R. D., Akimov, A. V. \& Aikens, C. M. Theoretical investigation of electron and nuclear dynamics in the [Au25(SH)18]-1 thiolate-protected gold nanocluster. J. Phys. Chem. C 121, 10653-10662 (2017).

51. Long, R., Liu, J. \& Prezhdo, O. V. Unravelling the effects of grain boundary and chemical doping on electron-hole recombination in $\mathrm{CH} 3 \mathrm{NH} 3 \mathrm{PbI} 3$ perovskite by time-domain atomistic simulation. J. Am. Chem. Soc. 138 3884-3890 (2016)

52. Long, R., Fang, W. H. \& Akimov, A. V. Nonradiative electron-hole recombination rate is greatly reduced by defects in monolayer black phosphorus: ab initio time domain study. J. Phys. Chem. Lett. 7, 653-659 (2016).

53. Giannozzi, P. et al. Quantum Espresso: a modular and open-source software project for quantum simulations of materials. J. Phys. Condens. Matter 21, 39 (2009).

54. Perdew, J. P., Burke, K. \& Ernzerhof, M. Generalized gradient approximation made simple. Phys. Rev. Lett. 77, 3865-3868 (1996).

55. Pradhan, E., Magyar, R. J. \& Akimov, A. V. Scaling relationships for nonadiabatic energy relaxation times in warm dense matter: toward understanding the equation of state. Phys. Chem. Chem. Phys. 18, 32466-32476 (2016).

56. Jaeger, H. M., Fischer, S. \& Prezhdo, O. V. Decoherence-induced surface hopping. J. Chem. Phys. 137, 22 A545 (2012)

57. Kresse, G. \& Furthmuller, J. Efficient iterative schemes for ab initio totalenergy calculations using a plane-wave basis set. Phys. Rev. B 54, 11169-11186 (1996).

58. Kresse, G. \& Furthmuller, J. Efficiency of ab-initio total energy calculations for metals and semiconductors using a plane-wave basis set. Comp. Mater. Sci. 6 15-50 (1996).

\section{Acknowledgements}

This work was supported by IBS-R004. This work was supported by "Next Generation Carbon Upcycling Project" (Project Number 2017M1A2A2046736) through the Nationa Research Foundation (NRF) funded by the Ministry of Science and ICT, Republic of Korea and KRICT. This work was supported by the Ministry of Science, ICT and Future Planning (MSIP) through the National Research Foundation of Korea (NRF) (NRF-2015M1A2A2053003). This work was also conducted under the framework of the Research and Development Program of the Korea Institute of Energy Research (KIER, B9-2434-03).

\section{Author contributions}

T.W.K., S.J., H.K., J.K., J.-O.B., and H.I. conceived the project. T.W.K. and I.O. carried out the spectroscopic experiment and analyzed the data with the help of 
S.J. and J.K. R.K.Y. and A.K. synthesized the sample. T.W.K., R.K.Y., A.K., C.-Y.Y., J.W.S., and R.R. performed the characterization of sample. Y.H., H.-K.L., and H.K. contributed to the theoretical study. T.W.K., Y.H., H.K., J.K., J.-O.B., and H.I. wrote the paper. All co-authors discussed the results and commented on the manuscript.

\section{Additional information}

Supplementary Information accompanies this paper at https://doi.org/10.1038/s41467019-09872-w.

Competing interests: The authors declare no competing interests.

Reprints and permission information is available online at http://npg.nature.com/ reprintsandpermissions/

Journal peer review information: Nature Communications thanks Abderrazzak Douhal, and other anonymous reviewer(s) for their contribution to the peer review of this work. Peer reviewer reports are available.
Publisher's note: Springer Nature remains neutral with regard to jurisdictional claims in published maps and institutional affiliations.

(c) (i) Open Access This article is licensed under a Creative Commons Attribution 4.0 International License, which permits use, sharing, adaptation, distribution and reproduction in any medium or format, as long as you give appropriate credit to the original author(s) and the source, provide a link to the Creative Commons license, and indicate if changes were made. The images or other third party material in this article are included in the article's Creative Commons license, unless indicated otherwise in a credit line to the material. If material is not included in the article's Creative Commons license and your intended use is not permitted by statutory regulation or exceeds the permitted use, you will need to obtain permission directly from the copyright holder. To view a copy of this license, visit http://creativecommons.org/ licenses/by/4.0/.

(c) The Author(s) 2019 